\title{
GENERALIZED RELAXED PROXIMAL POINT ALGORITHMS INVOLVING RELATIVE MAXIMAL ACCRETIVE MODELS WITH APPLICATIONS IN BANACH SPACES
}

\author{
RAM U. VERMA
}

\begin{abstract}
General models for the relaxed proximal point algorithm using the notion of relative maximal accretiveness (RMA) are developed, and then the convergence analysis for these models in the context of solving a general class of nonlinear inclusion problems differs significantly than that of Rockafellar (1976), where the local Lipschitz continuity at zero is adopted instead. Moreover, our approach not only generalizes convergence results to real Banach space settings, but also provides a suitable alternative to other problems arising from other related fields.
\end{abstract}

\section{Introduction}

Let $X$ be a real Banach space with $X^{*}$, the dual space of $X$. Let $\|\cdot\|$ denote the norm on $X$ and $X^{*}$, and let $\langle\cdot, \cdot\rangle$ denote the duality pairing between $X$ and $X^{*}$. We consider the inclusion problem: find a solution to

$$
0 \in M(g(x)),
$$

where $M: X \rightarrow 2^{X}$ is a set-valued mapping on $X$, and $g: X \rightarrow X$ is a single-valued mapping on $\mathrm{X}$ such that $\operatorname{range}(g) \cap \operatorname{dom}(M) \neq \emptyset$.

Just recently, the author [15] based on the work of Eckstein and Bertsekas [2] generalized the relaxed version of the proximal point algorithm and has shown that the sequence converges linearly to a solution of (1). On applying a local Lipschitz condition on the mapping $M^{-1}$ and with a more strengthened error tolerance, a convergence rate was obtained for the ordinary proximal point algorithm by Rockafellar [10], while Eckstein and Bertsekas [2] introduced a more relaxed version of the proximal point algorithm and applied the obtained results to the Douglas-Rachford splitting method for finding the zero of the sum of two monotone operators. Motivated by these algorithmic developments, we

Received June 1, 2009; Revised September 19, 2009.

2000 Mathematics Subject Classification. 49J40, 65B05.

Key words and phrases. variational inclusions, maximal relaxed accretive mapping, relative maximal accretive mapping, generalized resolvent operator. 
generalize the relaxed proximal point algorithm based on the notion of relative maximal accretiveness [1] for solving general inclusion problems in Banach space settings. This notion generalizes the general theory of maximal accretive set-valued mappings in a Banach space setting. In order to achieve local convergence, our approach differs significantly than that of Rockafellar [10], where the local Lipschitz type condition on the mapping $M^{-1}$ is imposed to derive the convergence rate estimate.

In this communication, our aim is to introduce relative maximal accretiveness (RMA) models and then apply them to approximation solvability of variational inclusion problems of the form (1) in a real Banach space setting. Unlike other existing notions, RMA models are applicable to other problems arising from several other fields, such as equilibria problems in economics, applied optimization and control theory, operations research, mathematical finance, management and decision sciences, and mathematical programming. For more details on the resolvent operator technique and its applications, and further developments, we refer the reader [1-38].

\section{Relative maximal accretiveness (RMA)}

In this section we discuss some results based on the basic properties and auxiliary results on relative maximal accretiveness. Let $X$ be a real Banach space and $X^{*}$ be the dual space of $X$. Let $\|\cdot\|$ denote the norm on $X$ and $X^{*}$, and let $\langle\cdot, \cdot\rangle$ denote the duality pairing between $X$ and $X^{*}$. Let $M: X \rightarrow 2^{X}$ be a multivalued mapping on $X$. We shall denote both the map $M$ and its graph by $M$, that is, the set $\{(x, y): y \in M(x)\}$. This is equivalent to stating that a mapping is any subset $M$ of $X \times X$, and $M(x)=\{y:(x, y) \in M\}$. If $M$ is single-valued, we shall still use $M(x)$ to represent the unique $y$ such that $(x, y) \in M$ rather than the singleton set $\{y\}$. This interpretation shall much depend on the context. The domain of a map $M$ is defined (as its projection onto the first argument) by

$$
D(M)=\{x \in X: \exists y \in X:(x, y) \in M\}=\{x \in X: M(x) \neq \emptyset\} .
$$

$D(M)=X$, shall denote the full domain of $M$, and the range of $M$ is defined by

$$
R(M)=\{y \in X: \exists x \in X:(x, y) \in M\} .
$$

The inverse $M^{-1}$ of $M$ is $\{(y, x):(x, y) \in M\}$. For a real number $\rho$ and a mapping $M$, let $\rho M=\{x, \rho y):(x, y) \in M\}$. If $L$ and $M$ are any mappings, we define

$$
L+M=\{(x, y+z):(x, y) \in L,(x, z) \in M\} .
$$

As we prepare for basic notions, we start with the generalized duality map$\operatorname{ping} J_{q}: X \rightarrow 2^{X^{*}}$

$$
J_{q}(x)=\left\{f^{*} \in X^{*}:\left\langle x, f^{*}\right\rangle=\|x\|^{q},\left\|f^{*}\right\|=\|x\|^{q-1}\right\} \quad \forall x \in X,
$$


where $q>1$. As a special case, $J_{2}$ is the normalized duality mapping, and $J_{q}(x)=\|x\|^{q-2} J_{2}(x)$ for $x \neq 0$. Next, as we head to uniformly smooth Banach spaces, we define the modulus of smoothness $\rho_{X}:[0, \infty) \rightarrow[0, \infty)$ by

$$
\rho_{X}(t)=\sup \left\{\frac{1}{2}(\|x+y\|+\|x-y\|)-1:\|x\| \leq 1,\|y\| \leq t\right\} .
$$

A Banach space $X$ is uniformly smooth if

$$
\lim _{t \rightarrow 0} \frac{\rho_{X}(t)}{t}=0
$$

and $X$ is $q$-uniformly smooth if there is a positive constant $c$ such that

$$
\rho_{X}(t) \leq c t^{q}, q>1 \text {. }
$$

Note that $J_{q}$ is single-valued if $\mathrm{X}$ is uniformly smooth. In this context, we state the following lemma from $\mathrm{Xu}$ [34].

Lemma 2.1 ([34]). Let $X$ be a uniformly smooth Banach space. Then $X$ is $q$-uniformly smooth if there exists a positive constant $c_{q}$ such that

$$
\|x+y\|^{q} \leq\|x\|^{q}+q\left\langle y, J_{q}(x)\right\rangle+c_{q}\|y\|^{q},
$$

where $q>1$.

Lemma 2.2. For any two nonnegative real numbers $a$ and $b$, we have

$$
(a+b)^{q} \leq 2^{q}\left(a^{q}+b^{q}\right) \text { for } q>1 .
$$

Definition 2.1. Let $M: X \rightarrow 2^{X}$ be a multivalued mapping on $X$ and $q>1$. The map $M$ is said to be:

(i) accretive if

$$
\left\langle u^{*}-v^{*}, J_{q}(u-v)\right\rangle \geq 0 \forall\left(u, u^{*}\right),\left(v, v^{*}\right) \in M .
$$

(ii) $(r)$-strongly accretive if there exists a positive constant $r$ such that

$$
\left\langle u^{*}-v^{*}, J_{q}(u-v)\right\rangle \geq r\|u-v\|^{q} \forall\left(u, u^{*}\right),\left(v, v^{*}\right) \in M .
$$

(iii) $(m)$-relaxed accretive if there exists a positive constant $\mathrm{m}$ such that

$$
\left\langle u^{*}-v^{*}, J_{q}(u-v)\right\rangle \geq(-m)\|u-v\|^{q} \forall\left(u, u^{*}\right),\left(v, v^{*}\right) \in M .
$$

(iv) (c)-cocoercively accretive if there exists a positive constant $\mathrm{c}$ such that

$$
\left\langle u^{*}-v^{*}, J_{q}(u-v)\right\rangle \geq c\left\|u^{*}-v^{*}\right\|^{q} \forall\left(u, u^{*}\right),\left(v, v^{*}\right) \in M .
$$

Definition 2.2. Let $A, B: X \rightarrow X$ be single-valued mappings on $X$ and $q>1$. The map $A$ is said to be:

(i) $(\gamma)$-cocoercively accretive with respect to $B$ if there exists a positive constant $\gamma$ such that

$$
\left\langle B(u)-B(v), J_{q}(A(u)-A(v))\right\rangle \geq \gamma\|A(u)-A(v)\|^{q} \forall u, v \in X .
$$

(ii) cocoercively accretive with respect to $B$ if

$$
\left\langle B(u)-B(v), J_{q}(A(u)-A(v))\right\rangle \geq\|A(u)-A(v)\|^{q} \forall u, v \in X .
$$


(iii) $(\gamma)$-cocoercively accretive if

$$
\left\langle u-v, J_{q}(A(u)-A(v))\right\rangle \geq \gamma\|A(u)-A(v)\|^{q} \forall u, v \in X .
$$

(iv) cocoercively accretive if

$$
\left\langle u-v, J_{q}(A(u)-A(v))\right\rangle \geq\|A(u)-A(v)\|^{q} \forall u, v \in X .
$$

Definition 2.3. Let $A: X \rightarrow X$ be a single-valued mapping. The map $M: X \rightarrow 2^{X}$ is said to be relative maximal accretive if

(i) $M$ is relative accretive (with respect to $A$ ), that is,

$$
\left\langle u^{*}-v^{*}, J_{q}(A(u)-A(v))\right\rangle \geq 0 \forall\left(u, u^{*}\right),\left(v, v^{*}\right) \in M .
$$

(ii) $R(I+\rho M)=X$ for $\rho>0$.

Example 2.1. Let $X=(-\infty,+\infty), M(x)=-x$ and $A(x)=-\frac{1}{2} x$. Then $M$ is relative monotone (with respect to $A$ ) but not monotone.

Example 2.2. Let $X$ be a real Hilbert space, and let $M: X \rightarrow 2^{X}$ be a maximal monotone mapping on $X$. Let $M_{\rho}=\rho^{-1}\left(I-R_{\rho}^{M}\right)$ denote the Yosida approximation of $M$, and $R_{\rho}^{M}=(I+\rho M)^{-1}$ denote the corresponding resolvent of $M$. Then for all $u, v \in X$,

$$
M_{\rho}(u) \in M\left(R_{\rho}^{M}(u)\right) \text { and } M_{\rho}(v) \in M\left(R_{\rho}^{M}(v)\right) .
$$

Since $M$ is maximal monotone, we have

$$
\left\langle M_{\rho}(u)-M_{\rho}(v), R_{\rho}^{M}(u)-R_{\rho}^{M}(v)\right\rangle \geq 0 \text { for } \rho>0 .
$$

Thus, $M_{\rho}$ is relative monotone (with respect to $R_{\rho}^{M}$ ).

Definition 2.4. Let $A: X \rightarrow X$ be an $(r)$-strongly accretive mapping and let $M: X \rightarrow 2^{X}$ be a relative maximal accretive mapping. Then the relative resolvent operator $J_{\rho, A}^{M}: X \rightarrow X$ is defined by

$$
J_{\rho, A}^{M}(u)=(I+\rho M)^{-1}(u) \text { for } \rho>0 .
$$

Proposition 2.1. Let $A: X \rightarrow X$ be an $(r)$-strongly monotone mapping and let $M: X \rightarrow 2^{X}$ be a relative maximal monotone mapping. Then the operator $(I+\rho M)^{-1}$ is single-valued.

\section{Generalized relaxed proximal point algorithm}

This section deals with an introduction of a generalized version of the relaxed proximal point algorithm and its applications to approximation solvability of the inclusion problem (1) based on the relative maximal accretiveness.

Lemma 3.1. Let $X$ be a real Banach space, let $A: X \rightarrow X$ be $(r)$-strongly accretive, and let $M: X \rightarrow 2^{X}$ be relative maximal accretive. In addition, if we suppose that

$\left\langle J_{\rho, A}^{M}(u)-J_{\rho, A}^{M}(v), J_{q}\left(A\left(J_{\rho, A}^{M}(u)\right)-A\left(J_{\rho, A}^{M}(v)\right)\right)\right\rangle \geq\left\|A\left(J_{\rho, A}^{M}(u)\right)-A\left(J_{\rho, A}^{M}(v)\right)\right\|^{q}$, 
then the corresponding resolvent operator associated with $M$ and defined by

$$
J_{\rho, A}^{M}(u)=(I+\rho M)^{-1}(u) \forall u \in X,
$$

is $\left(\frac{1}{r}\right)$-Lipschitz continuous, where $q>1$.

Proof. The proof follows from the definition of the resolvent operator

$$
J_{\rho, A}^{M}(u)=(I+\rho M)^{-1} .
$$

For any elements $u, v \in X$, we have

$$
\rho^{-1}\left[u-J_{\rho, A}^{M}(u)\right] \in M\left(J_{\rho, A}^{M}(u)\right)
$$

and

$$
\rho^{-1}\left[v-J_{\rho, A}^{M}(v)\right] \in M\left(J_{\rho, A}^{M}(v)\right) .
$$

Since $M$ is relative maximal accretive (with respect to $A$ ), we have

$$
\left\langle u-v-\left(J_{\rho, A}^{M}(u)-J_{\rho, A}^{M}(v)\right), J_{q}\left(A\left(J_{\rho, A}^{M}(u)\right)-A\left(J_{\rho, A}^{M}(v)\right)\right)\right\rangle \geq 0 .
$$

It follows that

$$
\begin{aligned}
& \left\langle u-v, J_{q}\left(A\left(J_{\rho, A}^{M}(u)\right)-A\left(J_{\rho, A}^{M}(v)\right)\right)\right\rangle \\
\geq & \left\langle J_{\rho, A}^{M}(u)-J_{\rho, A}^{M}(v), J_{q}\left(A\left(J_{\rho, A}^{M}(u)\right)-A\left(J_{\rho, A}^{M}(v)\right)\right)\right\rangle \\
\geq & \left\|A\left(J_{\rho, A}^{M}(u)\right)-A\left(J_{\rho, A}^{M}(v)\right)\right\|^{q} .
\end{aligned}
$$

Lemma 3.2. Let $X$ be a real Banach space, let $A: X \rightarrow X$ be $(r)$-strongly accretive, and let $M: X \rightarrow 2^{X}$ be relative maximal accretive. In addition, suppose that

$$
\begin{aligned}
& \left\langle J_{\rho, A}^{M}(u)-J_{\rho, A}^{M}(v), J_{q}\left(A\left(J_{\rho, A}^{M}(u)\right)-A\left(J_{\rho, A}^{M}(v)\right)\right)\right\rangle \\
\geq & \gamma\left\|A\left(J_{\rho, A}^{M}(u)\right)-A\left(J_{\rho, A}^{M}(v)\right)\right\|^{q} \text { for } \gamma>0,
\end{aligned}
$$

where $q>1$. Then the generalized resolvent operator associated with $M$ and defined by

$$
J_{\rho, A}^{M}(u)=(I+\rho M)^{-1}(u) \forall u \in X
$$

satisfies

$$
\left\|J_{\rho, A}^{M}(u)-J_{\rho, A}^{M}(v)\right\| \leq \frac{1}{\gamma r}\|u-v\|
$$

where $\gamma>0$

Proof. The proofs is quite similar to that of Lemma 3.1. Since $A$ is $(r)$-strongly accretive (and hence $\|A(u)-A(v)\| \geq r\|u-v\|$ ), we have

$$
\|u-v\| \geq \gamma\left\|A\left(J_{\rho, A}^{M}(u)\right)-A\left(J_{\rho, A}^{M}(v)\right)\right\|,
$$

or

$$
\|u-v\| \geq \gamma r\left\|J_{\rho, A}^{M}(u)-J_{\rho, A}^{M}(v)\right\| .
$$


Remark 3.1. Note that this is a new class of mappings that satisfies

$$
\begin{aligned}
& \left\langle J_{\rho, A}^{M}(u)-J_{\rho, A}^{M}(v), J_{q}\left(A\left(J_{\rho, A}^{M}(u)\right)-A\left(J_{\rho, A}^{M}(v)\right)\right)\right\rangle \\
\geq & \gamma\left\|A\left(J_{\rho, A}^{M}(u)\right)-A\left(J_{\rho, A}^{M}(v)\right)\right\|^{q} \text { for } \gamma>0,
\end{aligned}
$$

in a Banach space setting, but it coincides with the notions of the relative cocoercivity or just cocoercivity in a Hilbert space setting, for example, in following Lemmas 3.3 and 3.4.

Lemma 3.3. Let $X$ be a real Hilbert space, let $A: X \rightarrow X$ be $(r)$-strongly monotone and $(\gamma)$-cocoercive, that is,

$$
\langle A(u)-A(v), u-v\rangle \geq \gamma\|A(u)-A(v)\|^{2} \text { for } u, v \in X,
$$

and let $M: X \rightarrow 2^{X}$ be relative maximal monotone. Then the generalized resolvent operator associated with $M$ and defined by

$$
J_{\rho, A}^{M}(u)=(I+\rho M)^{-1}(u) \forall u \in X,
$$

satisfies

where $\gamma>0$

$$
\left\|J_{\rho, A}^{M}(u)-J_{\rho, A}^{M}(v)\right\| \leq \frac{1}{\gamma r}\|u-v\|
$$

Proof. The proof follows from the definition of the resolvent operator

$$
J_{\rho, A}^{M}(u)=(I+\rho M)^{-1} .
$$

For any elements $u, v \in X$, we have

$$
\rho^{-1}\left[u-J_{\rho, A}^{M}(u)\right] \in M\left(J_{\rho, A}^{M}(u)\right)
$$

and

$$
\rho^{-1}\left[v-J_{\rho, A}^{M}(v)\right] \in M\left(J_{\rho, A}^{M}(v)\right) .
$$

Since $M$ is relative maximal monotone (with respect to $A$ ), we have

$$
\left\langle u-v-\left(J_{\rho, A}^{M}(u)-J_{\rho, A}^{M}(v)\right), A\left(J_{\rho, A}^{M}(u)\right)-A\left(J_{\rho, A}^{M}(v)\right)\right\rangle \geq 0 .
$$

It follows that

$$
\begin{aligned}
& \left\langle u-v, A\left(J_{\rho, A}^{M}(u)\right)-A\left(J_{\rho, A}^{M}(v)\right)\right\rangle \\
\geq & \left\langle J_{\rho, A}^{M}(u)-J_{\rho, A}^{M}(v), A\left(J_{\rho, A}^{M}(u)\right)-A\left(J_{\rho, A}^{M}(v)\right)\right\rangle \\
\geq & \gamma\left\|A\left(J_{\rho, A}^{M}(u)\right)-A\left(J_{\rho, A}^{M}(v)\right)\right\|^{2} .
\end{aligned}
$$

Lemma 3.4. Let $X$ be a real Hilbert space, and let $M: X \rightarrow 2^{X}$ be maximal monotone. Then the resolvent operator associated with $M$ and defined by

$$
R_{\rho}^{M}(u)=(I+\rho M)^{-1}(u) \forall u \in X,
$$

satisfies

where $\rho>0$.

$$
\left\|R_{\rho}^{M}(u)-R_{\rho}^{M}(v)\right\| \leq\|u-v\|,
$$


Now we focus our attention to establish the main results on the relative maximal accretivity (RMA) relating to the approximation solvability of (1).

Theorem 3.1. Let $X$ be a real Banach space, let $A: X \rightarrow X$ be $(r)$-strongly accretive, and let $M: X \rightarrow 2^{X}$ be relative maximal accretive. Let $g: X \rightarrow X$ be a map on $X$. Then the following statements are equivalent:

(i) An element $u \in X$ is a solution to (1).

(ii) For an $u \in X$, we have

$$
g(u)=J_{\rho, A}^{M}(g(u)),
$$

where

$$
J_{\rho, A}^{M}(u)=(I+\rho M)^{-1}(u) \text { for } \rho>0 .
$$

In the following theorem, we apply the generalized relaxed proximal point algorithm to approximate the solution of (1), and as a result, we achieve linear convergence.

Theorem 3.2. Let $X$ be a real q-uniformly smooth Banach space, let $A: X \rightarrow$ $X$ be $(r)$-strongly accretive, let $g: X \rightarrow X$ be $(c)$-strongly accretive, and let $M: X \rightarrow 2^{X}$ be relative maximal accretive $(R M A)$. Furthermore, suppose that

$$
\begin{aligned}
& \left\langle J_{\rho, A}^{M}(g(u))-J_{\rho, A}^{M}(g(v)), J_{q}\left(A\left(J_{\rho, A}^{M}(g(u))\right)-A\left(J_{\rho, A}^{M}(g(v))\right)\right)\right\rangle \\
\geq & \gamma\left\|A\left(J_{\rho, A}^{M}(g(u))\right)-A\left(J_{\rho, A}^{M}(g(v))\right)\right\|^{q} \text { for } \gamma>0,
\end{aligned}
$$

where $q>1$.

For an arbitrarily chosen initial point $x^{0}$, suppose that the sequence $\left\{g\left(x^{k}\right)\right\}$ is generated by the generalized proximal point algorithm

$$
g\left(x^{k+1}\right)=\left(1-\alpha_{k}\right) g\left(x^{k}\right)+\alpha_{k} y^{k} \forall k \geq 0,
$$

and $y^{k}$ satisfies

$$
\left\|y^{k}-J_{\rho, A}^{M}\left(g\left(x^{k}\right)\right)\right\| \leq \delta_{k}\left\|y^{k}-g\left(x^{k}\right)\right\|,
$$

where

$$
J_{\rho, A}^{M}=(I+\rho M)^{-1}
$$

and

$$
\left\{\delta_{k}\right\},\left\{\alpha_{k}\right\} \subseteq[0, \infty)
$$

are scalar sequences. Then the sequence $\left\{x^{k}\right\}$ converges linearly to a solution of (1) with the convergence rate

$$
\limsup \frac{\theta_{k}+\delta_{k}}{1-\delta_{k}}=\limsup \theta_{k}=\left[1-\triangle\left(1-\frac{1}{\gamma r}\right)\right]<1,
$$

where $\gamma r>1, \sum_{k=0}^{\infty} \delta_{k}<\infty, \delta_{k} \rightarrow 0, \alpha_{k} \leq 1, \triangle=\inf \alpha_{k}>0$, and $\alpha=$ $\limsup _{k \rightarrow \infty} \alpha_{k}$. 
Proof. Suppose that $x^{*}$ is a zero of $M$. From Theorem 3.1, it follows that any solution to (1) is a fixed point of $J_{\rho, A}^{M}$. Since $A$ is $(r)$-strongly accretive (and hence $\|A(u)-A(v)\| \geq r\|u-v\|)$ and using Lemma 3.2, we have a crucial inequality

$$
\left\|J_{\rho, A}^{M}\left(g\left(x^{k}\right)\right)-J_{\rho, A}^{M}\left(g\left(x^{*}\right)\right)\right\| \leq \frac{1}{\gamma r}\left\|g\left(x^{k}\right)-g\left(x^{*}\right)\right\| .
$$

Next, for all $k \geq 0$, we express

$$
g\left(z^{k+1}\right)=\left(1-\alpha_{k}\right) g\left(x^{k}\right)+\alpha_{k} J_{\rho, A}^{M}\left(g\left(x^{k}\right)\right) .
$$

Then we find the estimate using (3) that

$$
\begin{aligned}
& \left\|g\left(z^{k+1}\right)-g\left(x^{*}\right)\right\|=\left\|\left(1-\alpha_{k}\right) g\left(x^{k}\right)+\alpha_{k} J_{\rho, A}^{M}\left(g\left(x^{k}\right)\right)-g\left(x^{*}\right)\right\| \\
= & \left\|\left(1-\alpha_{k}\right)\left(g\left(x^{k}\right)-g\left(x^{*}\right)\right)+\alpha_{k}\left(J_{\rho, A}^{M}\left(g\left(x^{k}\right)\right)-J_{\rho, A}^{M}\left(g\left(x^{*}\right)\right)\right)\right\| \\
\leq & \left(1-\alpha_{k}\right)\left\|g\left(x^{k}\right)-g\left(x^{*}\right)\right\|+\alpha_{k}\left\|J_{\rho, A}^{M}\left(g\left(x^{k}\right)\right)-J_{\rho, A}^{M}\left(g\left(x^{*}\right)\right)\right\| \\
\leq & {\left[\left(1-\alpha_{k}\right)+\frac{\alpha_{k}}{\gamma r}\right]\left\|g\left(x^{k}\right)-g\left(x^{*}\right)\right\| } \\
= & {\left[1-\alpha_{k}\left(1-\frac{1}{\gamma r}\right)\right]\left\|g\left(x^{k}\right)-g\left(x^{*}\right)\right\| } \\
\leq & {\left[1-\triangle\left(1-\frac{1}{\gamma r}\right)\right]\left\|g\left(x^{k}\right)-g\left(x^{*}\right)\right\| } \\
= & \theta_{k}\left\|g\left(x^{k}\right)-g\left(x^{*}\right)\right\|,
\end{aligned}
$$

where $\theta_{k}=\left[1-\triangle\left(1-\frac{1}{\gamma r}\right)\right]$ and $\gamma r>1$. Thus, we have

$$
\left\|g\left(z^{k+1}\right)-g\left(x^{*}\right)\right\| \leq \theta_{k}\left\|g\left(x^{k}\right)-g\left(x^{*}\right)\right\| .
$$

Since $g\left(x^{k+1}\right)=\left(1-\alpha_{k}\right) g\left(x^{k}\right)+\alpha_{k} y^{k}$, we have

$$
g\left(x^{k+1}\right)-g\left(x^{k}\right)=\alpha_{k}\left(y^{k}-g\left(x^{k}\right)\right) .
$$

On the other hand, we have

$$
\begin{aligned}
& \left\|g\left(x^{k+1}\right)-g\left(z^{k+1}\right)\right\| \\
= & \left\|\left(1-\alpha_{k}\right) g\left(x^{k}\right)+\alpha_{k} y^{k}-\left[\left(1-\alpha_{k}\right) g\left(x^{k}\right)+\alpha_{k} J_{\rho, A}^{M}\left(g\left(x^{k}\right)\right)\right]\right\| \\
= & \left\|\alpha_{k}\left(y^{k}-J_{\rho, A}^{M}\left(g\left(x^{k}\right)\right)\right)\right\| \\
\leq & \alpha_{k} \delta_{k}\left\|y^{k}-g\left(x^{k}\right)\right\| .
\end{aligned}
$$

Finally, we estimate using the above arguments that

$$
\begin{aligned}
& \left\|g\left(x^{k+1}\right)-g\left(x^{*}\right)\right\| \\
\leq & \left\|g\left(z^{k+1}\right)-g\left(x^{*}\right)\right\|+\left\|g\left(x^{k+1}\right)-g\left(z^{k+1}\right)\right\| \\
\leq & \left\|g\left(z^{k+1}\right)-g\left(x^{*}\right)\right\|+\alpha_{k} \delta_{k}\left\|y^{k}-g\left(x^{k}\right)\right\| \\
\leq & \left\|g\left(z^{k+1}\right)-g\left(x^{*}\right)\right\|+\delta_{k}\left\|g\left(x^{k+1}\right)-g\left(x^{k}\right)\right\| \\
\leq & \left\|g\left(z^{k+1}\right)-g\left(x^{*}\right)\right\|+\delta_{k}\left\|g\left(x^{k+1}\right)-g\left(x^{*}\right)\right\|+\delta_{k}\left\|g\left(x^{k}\right)-g\left(x^{*}\right)\right\| .
\end{aligned}
$$


Thus, we conclude that

$$
\begin{aligned}
& \left(1-\delta_{k}\right)\left\|g\left(x^{k+1}\right)-g\left(x^{*}\right)\right\| \\
\leq & \left\|g\left(z^{k+1}\right)-g\left(x^{*}\right)\right\|+\delta_{k}\left\|g\left(x^{k}\right)-g\left(x^{*}\right)\right\| \\
\leq & \theta_{k}\left\|g\left(x^{k}\right)-g\left(x^{*}\right)\right\|+\delta_{k}\left\|g\left(x^{k}\right)-g\left(x^{*}\right)\right\| \\
= & \left(\theta_{k}+\delta_{k}\right)\left\|g\left(x^{k}\right)-g\left(x^{*}\right)\right\|,
\end{aligned}
$$

so we have

$$
\left\|g\left(x^{k+1}\right)-g\left(x^{*}\right)\right\| \leq \frac{\theta_{k}+\delta_{k}}{1-\delta_{k}}\left\|g\left(x^{k}\right)-g\left(x^{*}\right)\right\| .
$$

Hence, $\left\{g\left(x^{k}\right)\right\}$ converges to $g\left(x^{*}\right)$.

Finally, since $g$ is $(c)$-strongly accretive (and hence, $\|g(x)-g(y)\| \geq c\|x-y\|$ ), we conclude that the sequence $\left\{x^{k}\right\}$ converges to $x^{*}$.

For $\gamma=1$ in Theorem 3.2, we have:

Theorem 3.3. Let $X$ be a real q-uniformly smooth Banach space, let $A$ : $X \rightarrow X$ be $(r)$-strongly accretive, and let $M: X \rightarrow 2^{X}$ be RMA. Furthermore, suppose

$$
\begin{aligned}
& \left\langle J_{\rho, A}^{M}(u)-J_{\rho, A}^{M}(v), J_{q}\left(A\left(J_{\rho, A}^{M}(u)\right)-A\left(J_{\rho, A}^{M}(v)\right)\right)\right\rangle \\
\geq & \left\|A\left(J_{\rho, A}^{M}(u)\right)-A\left(J_{\rho, A}^{M}(v)\right)\right\|^{q} \text { for } \gamma>0,
\end{aligned}
$$

where $q>1$.

For an arbitrarily chosen initial point $x^{0}$, suppose that the sequence $\left\{x^{k}\right\}$ is generated by the generalized proximal point algorithm

$$
g\left(x^{k+1}\right)=\left(1-\alpha_{k}\right) g\left(x^{k}\right)+\alpha_{k} y^{k} \forall k \geq 0,
$$

and $y^{k}$ satisfies

$$
\left\|y^{k}-J_{\rho, A}^{M}\left(g\left(x^{k}\right)\right)\right\| \leq \delta_{k}\left\|y^{k}-g\left(x^{k}\right)\right\|
$$

where

$$
J_{\rho_{k}}^{M}=(I+\rho M)^{-1}
$$

and

$$
\left\{\delta_{k}\right\},\left\{\alpha_{k}\right\} \subseteq[0, \infty)
$$

are scalar sequences. Then the sequence $\left\{x^{k}\right\}$ converges linearly to a solution of (1) with the convergence rate

$$
\theta_{k}=\left[1-\triangle\left(1-\frac{1}{r}\right)\right]<1,
$$

where $r>1, \sum_{k=0}^{\infty} \delta_{k}<\infty, \delta_{k} \rightarrow 0, \triangle=\inf \alpha_{k}>0$, and $\alpha=\lim \sup _{k \rightarrow \infty} \alpha_{k}$.

When $g=I$, Theorem 3.2 reduces to the approximation solvability of the inclusion problem: determine a solution to

$$
0 \in M(x) .
$$


Theorem 3.4. Let $X$ be a real q-uniformly smooth Banach space, let $A: X \rightarrow$ $X$ be $(r)$-strongly accretive, and let $M: X \rightarrow 2^{X}$ be relative maximal accretive $(R M A)$. Furthermore, suppose that

$$
\begin{aligned}
& \left\langle J_{\rho, A}^{M}(u)-J_{\rho, A}^{M}(v), J_{q}\left(A\left(J_{\rho, A}^{M}(u)\right)-A\left(J_{\rho, A}^{M}(v)\right)\right)\right\rangle \\
\geq & \gamma\left\|A\left(J_{\rho, A}^{M}(u)\right)-A\left(J_{\rho, A}^{M}(v)\right)\right\|^{q} \text { for } \gamma>0,
\end{aligned}
$$

where $q>1$.

For an arbitrarily chosen initial point $x^{0}$, suppose that the sequence $\left\{x^{k}\right\}$ is generated by the generalized proximal point algorithm

$$
x^{k+1}=\left(1-\alpha_{k}\right) x^{k}+\alpha_{k} y^{k} \quad \forall k \geq 0,
$$

and $y^{k}$ satisfies

$$
\left\|y^{k}-J_{\rho, A}^{M}\left(x^{k}\right)\right\| \leq \delta_{k}\left\|y^{k}-x^{k}\right\|,
$$

where

$$
J_{\rho, A}^{M}=(I+\rho M)^{-1}
$$

and

$$
\left\{\delta_{k}\right\},\left\{\alpha_{k}\right\} \subseteq[0, \infty)
$$

are scalar sequences. Then the sequence $\left\{x^{k}\right\}$ converges linearly to a solution of (9) with the convergence rate

$$
\theta_{k}=\left[1-\triangle\left(1-\frac{1}{\gamma r}\right)\right]<1,
$$

where $\gamma r>1, \sum_{k=0}^{\infty} \delta_{k}<\infty, \delta_{k} \rightarrow 0, \alpha_{k} \leq 1, \triangle=\inf \alpha_{k}>0$, and $\alpha=$ $\limsup _{k \rightarrow \infty} \alpha_{k}$.

Proof. Suppose that $x^{*}$ is a zero of $M$. From Theorem 3.1, it follows that any solution to (9) is a fixed point of $J_{\rho, A}^{M}$. Since $A$ is $(r)$-strongly accretive (and hence $\|A(u)-A(v)\| \geq r\|u-v\|)$ and using Lemma 3.2, we have the inequality

$$
\left\|J_{\rho, A}^{M}\left(x^{k}\right)-J_{\rho, A}^{M}\left(x^{*}\right)\right\| \leq \frac{1}{\gamma r}\left\|x^{k}-x^{*}\right\| .
$$

Next, for all $k \geq 0$, we express

$$
z^{k+1}=\left(1-\alpha_{k}\right) x^{k}+\alpha_{k} J_{\rho, A}^{M}\left(x^{k}\right) .
$$

Then we find the estimate using (11) that

$$
\begin{aligned}
& \left\|z^{k+1}-x^{*}\right\|=\left\|\left(1-\alpha_{k}\right) x^{k}+\alpha_{k} J_{\rho, A}^{M}\left(x^{k}\right)-x^{*}\right\| \\
= & \left\|\left(1-\alpha_{k}\right)\left(x^{k}-x^{*}\right)+\alpha_{k}\left(J_{\rho, A}^{M}\left(x^{k}\right)-J_{\rho, A}^{M}\left(x^{*}\right)\right)\right\| \\
\leq & \left(1-\alpha_{k}\right)\left\|x^{k}-x^{*}\right\|+\alpha_{k}\left\|J_{\rho, A}^{M}\left(x^{k}\right)-J_{\rho, A}^{M}\left(x^{*}\right)\right\| \\
\leq & {\left[\left(1-\alpha_{k}\right)+\frac{\alpha_{k}}{\gamma r}\right]\left\|x^{k}-x^{*}\right\| } \\
= & {\left[1-\alpha_{k}\left(1-\frac{1}{\gamma r}\right)\right]\left\|x^{k}-x^{*}\right\| }
\end{aligned}
$$




$$
\begin{aligned}
& \leq\left[1-\triangle\left(1-\frac{1}{\gamma r}\right)\right]\left\|x^{k}-x^{*}\right\| \\
& =\theta_{k}\left\|x^{k}-x^{*}\right\|,
\end{aligned}
$$

where $\theta_{k}=\left[1-\triangle\left(1-\frac{1}{\gamma r}\right)\right]$ and $\gamma r>1$. Thus, we have

$$
\left\|z^{k+1}-x^{*}\right\| \leq \theta_{k}\left\|x^{k}-x^{*}\right\| .
$$

Since $x^{k+1}=\left(1-\alpha_{k}\right) x^{k}+\alpha_{k} y^{k}$, we have $x^{k+1}-x^{k}=\alpha_{k}\left(y^{k}-x^{k}\right)$.

On the other hand, we have

$$
\begin{aligned}
& \left\|x^{k+1}-z^{k+1}\right\| \\
= & \left\|\left(1-\alpha_{k}\right) x^{k}+\alpha_{k} y^{k}-\left[\left(1-\alpha_{k}\right) x^{k}+\alpha_{k} J_{\rho, A}^{M}\left(x^{k}\right)\right]\right\| \\
= & \left\|\alpha_{k}\left(y^{k}-J_{\rho, A}^{M}\left(x^{k}\right)\right)\right\| \\
\leq & \alpha_{k} \delta_{k}\left\|y^{k}-x^{k}\right\| .
\end{aligned}
$$

It follows that

$$
\begin{aligned}
& \left\|x^{k+1}-x^{*}\right\| \\
\leq & \left\|z^{k+1}-x^{*}\right\|+\left\|x^{k+1}-z^{k+1}\right\| \\
\leq & \left\|z^{k+1}-x^{*}\right\|+\alpha_{k} \delta_{k}\left\|y^{k}-x^{k}\right\| \\
\leq & \left\|z^{k+1}-x^{*}\right\|+\delta_{k}\left\|x^{k+1}-x^{k}\right\| \\
\leq & \left\|z^{k+1}-x^{*}\right\|+\delta_{k}\left\|x^{k+1}-x^{*}\right\|+\delta_{k}\left\|x^{k}-x^{*}\right\| .
\end{aligned}
$$

Thus, we conclude that

$$
\begin{aligned}
& \left(1-\delta_{k}\right)\left\|x^{k+1}-x^{*}\right\| \\
\leq & \left\|z^{k+1}-x^{*}\right\|+\delta_{k}\left\|x^{k}-x^{*}\right\| \\
\leq & \theta_{k}\left\|x^{k}-x^{*}\right\|+\delta_{k}\left\|x^{k}-x^{*}\right\| \\
= & \left(\theta_{k}+\delta_{k}\right)\left\|x^{k}-x^{*}\right\|,
\end{aligned}
$$

so we have

$$
\left\|x^{k+1}-x^{*}\right\| \leq \frac{\theta_{k}+\delta_{k}}{1-\delta_{k}}\left\|x^{k}-x^{*}\right\| .
$$

Hence, $\left\{x^{k}\right\}$ converges to $x^{*}$.

\section{An application}

Let $X$ be a real Banach space and let $f: X \rightarrow R$ be a locally Lipschitz functional on $\mathrm{X}$. We consider the inclusion problem: determine a solution to

$$
0 \in \partial f(x),
$$

where $\partial f: X \rightarrow 2^{X}$ is a set-valued mapping on $X$. Then it turns out that $I+\partial f$ is relative accretive if $A: X \rightarrow X$ is $(r)$-strongly accretive, and $\partial f: X \rightarrow 2^{X}$ is relative accretive. This is equivalent to stating that $\partial f$ is relative maximal accretive. Now all the conditions for Theorem 3.2 are satisfied, and one can apply Theorem 3.2 to the solvability of (16). 
Acknowledgment. The author is greatly indebted to referees for their valuable comments and suggestions leading to the revised version.

\section{References}

[1] R. P. Agarwal and R. U. Verma, Role of relative A-maximal monotonicity in overrelaxed proximal-point algorithms with applications, J. Optim. Theory Appl. 143 (2009), no. 1, 1-15; doi 10.1017/s10957-009-9554-z.

[2] J. Eckstein and D. P. Bertsekas, On the Douglas-Rachford splitting method and the proximal point algorithm for maximal monotone operators, Math. Programming 55 (1992), no. 3, Ser. A, 293-318.

[3] Y. P. Fang and N. J. Huang, H-monotone operators and system of variational inclusions, Comm. Appl. Nonlinear Anal. 11 (2004), no. 1, 93-101.

[4] M. Fukushima, The primal Douglas-Rachford splitting algorithm for a class of monotone mappings with application to the traffic equilibrium problem, Math. Programming $\mathbf{7 2}$ (1996), no. 1, Ser. A, 1-15.

[5] R. Glowinski and P. Le Tellec, Augmented Lagrangian and Operator-Splitting Methods in Nonlinear Mechanics, SIAM Studies in Applied Mathematics, 9. Society for Industrial and Applied Mathematics (SIAM), Philadelphia, PA, 1989.

[6] H. Y. Lan, J. H. Kim, and Y. J. Cho, On a new system of nonlinear A-monotone multivalued variational inclusions, J. Math. Anal. Appl. 327 (2007), no. 1, 481-493.

[7] B. Martinet, Régularisation d'inéquations variationnelles par approximations successives, Rev. Francaise Inform. Rech. Oper. Ser. R-3 4 (1970), 154-158.

[8] A. Moudafi, Mixed equilibrium problems: sensitivity analysis and algorithmic aspect, Comput. Math. Appl. 44 (2002), no. 8-9, 1099-1108.

[9] S. M. Robinson, Composition duality and maximal monotonicity, Math. Program. 85 (1999), no. 1, Ser. A, 1-13.

[10] R. T. Rockafellar, Monotone operators and the proximal point algorithm, SIAM J. Control Optimization 14 (1976), no. 5, 877-898.

[11] _ Augmented Lagrangians and applications of the proximal point algorithm in convex programming, Math. Oper. Res. 1 (1976), no. 2, 97-116.

[12] R. T. Rockafellar and R. J.-B. Wets, Variational Analysis, Springer-Verlag, Berlin, 2004.

[13] P. Tseng, Alternating projection-proximal methods for convex programming and variational inequalities, SIAM J. Optim. 7 (1997), no. 4, 951-965.

[14] _ A modified forward-backward splitting method for maximal monotone mappings, SIAM J. Control Optim. 38 (2000), no. 2, 431-446.

[15] R. U. Verma, Rockafellar's theorem on linear convergence and A-proximal point algorithm, DCDIS: Dynamics of Continuous, Discrete and Impulsive Systems (in press).

[16] _ Sensitivity analysis for generalized strongly monotone variational inclusions based on the $(A, \eta)$-resolvent operator technique, Appl. Math. Lett. 19 (2006), no. 12, 1409-1413.

[17] _ A-monotonicity and its role in nonlinear variational inclusions, J. Optim. Theory Appl. 129 (2006), no. 3, 457-467.

[18] _ General system of A-monotone nonlinear variational inclusion problems with applications, J. Optim. Theory Appl. 131 (2006), no. 1, 151-157.

[19] _ A-monotone nonlinear relaxed cocoercive variational inclusions, Cent. Eur. J. Math. 5 (2007), no. 2, 386-396.

[20] _ General system of $(A, \eta)$-monotone variational inclusion problems based on generalized hybrid iterative algorithm, Nonlinear Anal. Hybrid Syst. 1 (2007), no. 3, 326-335.

[21] _ Approximation solvability of a class of nonlinear set-valued variational inclusions involving $(A, \eta)$-monotone mappings, J. Math. Anal. Appl. 337 (2008), no. 2, 969-975. 
[22] _ On a class of nonlinear variational inequalities involving partially relaxed monotone and partially strongly monotone mappings, Math. Sci. Res. Hot-Line 4 (2000), no. 2, 57-65.

[23] - Averaging techniques and cocoercively monotone mappings, Math. Sci. Res. J. 10 (2006), no. 3, 79-82.

[24] - Rockafellar's celebrated theorem based on A-maximal monotonicity design, Appl. Math. Lett. 21 (2008), no. 4, 355-360.

[25] - On the generalized proximal point algorithm with applications to inclusion problems, J. Ind. Manag. Optim. 5 (2009), no. 2, 381-390.

[26] _ General implicit variational inclusion problems involving A-maximal relaxed accretive mappings in Banach spaces, Archivum Mathematicum 45 (2009), no. 3, 171177.

[27] _ Generalized maximal monotonicity models and their applications to nonlinear variational inclusion problems and beyond, Int. J. Optim. Theory Methods Appl. 1 (2009), no. 2, 182-206.

[28] _ New proximal point algorithms based on RMM models for nonlinear variational inclusion problems, Adv. Nonlinear Var. Inequal. 12 (2009), no. 2, 91-102.

[29] _ Generalized first-order nonlinear evolution equations and generalized Yosida approximations based on $H$-maximal monotonicity frameworks, Electron. J. Differential Equations 2009 (2009), No. 85, 16 pp.

[30] _ A new relaxed proximal point procedure and applications to nonlinear variational inclusions, Comput. Math. Appl. 58 (2009), no. 8, 1631-1635.

[31] _ General class of implicit inclusion problems based on A-maximal relaxed monotone models with applications, Journal of Numerical Mathematics and Stochastics 1 (2009), no. 1, 24-32.

[32] _ The generalized relaxed proximal point algorithm involving A-maximal relaxed accretive mappings with applications to Banach spaces, Mathematical and Computer Modelling 50 (2009), no. 7-8, 1026-1032.

[33] H. K. Xu, Iterative algorithms for nonlinear operators, J. London Math. Soc. (2) 66 (2002), no. 1, 240-256.

[34] Inequalities in Banach spaces with applications, Nonlinear Anal. 16 (1991), no. 12, 1127-1138.

[35] E. Zeidler, Nonlinear Functional Analysis and its Applications I, Springer-Verlag, New York, 1986.

[36] _ Nonlinear Functional Analysis and its Applications II/A, Springer-Verlag, New York, 1990.

[37] _ Nonlinear Functional Analysis and its Applications II/B, Springer-Verlag, New York, New York, 1990.

[38] _ Nonlinear Functional Analysis and its Applications III, Springer-Verlag, New York, New York, 1984.

Florida Institute of TeChNOLOGy

Department of Mathematical Sciences

Melbourne, Florida 32901, USA

AND

International Publications (USA)

12085 Lake Cypress Circle

Suite I109

Orlando, Florida 32828, USA

E-mail address: verma99@msn.com 